\title{
ISOLATION OF CORTICAL MATERIAL FROM SEA URCHIN EGGS BY CENTRIFUGATION ${ }^{1}$
}

\author{
ROBERT DAY ALLEN ${ }^{2}$ \\ Department of Zoology, University of Michigan, Ann Arbor \\ EIGHT FIGURES
}

INTRODUCTION

Although the surface layers of the sea urchin egg are the site of dramatic changes at fertilization, little is actually known about the submicroscopic structure of the surface layers, their chemistry, or the real nature of the physiological changes that are collectively referred to as the cortical reaction.

Excluding the jelly, the surface layers of the unfertilized egg that have been recognized are (1) the vitelline membrane or its precursor, (2) the "luminous hyaline layer," which changes color at fertilization when viewed under dark-ground illumination (Runnström, '23), and (3) the "Harvey-Moser cortex" containing granules which ordinarily do not move under centrifugal force (F. N. Harvey, '11; Moser, '39). The plasma-membrane has not been directly observed and identified as a structure; however, recent evidence from permeability studies has tentatively localized this structure beneath the cortex (Parpart and Laris, '54). A similar conclusion has been reached on the basis of observations on eggs deprived of strips of cortical material; such eggs did not undergo cytolysis or lose any eytoplasm through rupture of the true cell surface (Allen, '55).

${ }^{2}$ This investigation was supported by a research grant (C-2609) from the National Caneer Institute of the National Institutes of Health, Public Health Service.

${ }^{2}$ Address after September 1, 1956 : Department of Biology, Princeton University, Princeton, New Jersey. 
Two methods have been reported for the isolation of cortical material. Broken hulls of cortex have been recovered after the centrifugation of homogenates of acid-fixed eggs (Motomura, '54). Strips of cortex were isolated by pulling away eggs adhering to glass; these strips responded to insemination (Allen, '55).

The recent realization that the cortex of the sea urchin egg might be an "extra-cellular" structure suggested the possibility that a specific gravity difference between cortex and the rest of the egg might render isolation of cortical material possible by centrifugation without rupture of the cell itself. Such a prospect would be attractive, for cortical material isolated in this way would be free from the criticism of impurity due to physical contamination or adsorption of endoplasmic substances.

Although the possibilities for chemical investigation of isolated cortical material are considerable, the present paper will be concerned only with details of methodology for cortical isolation, and with the structure of the isolated cortical layers.

MATERIAL AND METHODS

Eggs of the sea urchin Strongylocentrotus purpuratus were obtained from animals collected along the California coast, during late January and early February, 1956. Animals were kept in moist sacks in a $4^{\circ} \mathrm{C}$. cold room in Berkeley, where their gametes remained fertilizable for from 4 to 5 days.

Most experiments were performed on jelly-free eggs which had been treated with acid sea water ( $\mathrm{pH} 4.5)$ for two minutes. Jelly removal would be an important step toward ultimate chemical analysis of the isolated cortical material, for the jelly is sufficiently heavy to contaminate the bottom fractions.

Separation of whole, jelly-free egg's into halves and quarters was accomplished by layering the eggs over isotonic sucrose (0.96 molar) in plastic centrifuge tubes. These were tilted several times to form a broad gradient between the sea water and sucrose. Separation of light and heavy halves was obtained 
at $9,000 \times$ gravity for 10 minutes. Eggs subjected to 11,500 $X$ gravity yielded quarters in the same amount of time (fig. 1).

Isolation of cortical material from whole eggs, halves, or quarters was carried out either in the no. 40 angle-head, or in the new SW-39-L "swing-out bucket" rotor of the Spinco Preparative Ultracentrifuge. The SW-39-L rotor was superior in the resolution of layers obtainable. It receives tubes $1.27 \mathrm{~cm}$ in diameter and $5.4 \mathrm{~cm}$ long. The bottom centimeter was filled with hypertonic sucrose $(40-50 \%)$, over which was placed 0.96 molar sucrose (approximately isotonic). Eggs in sea water were layered on the sucrose from approximately half way up the tube to within a few millimeters of the top. The tubes were tilted several times to establish a broad density gradient. In some experiments the bottom layer of sucrose was omitted.

Low temperature facilitated fragmentation of the eggs by centrifugal force. Therefore, separation of halves and quarters (E. B. Harvey, '32) was performed in the cold. For the isolation of cortical material, however, it was desirable to centrifuge at room temperature in order that the cortex would be removed before the eggs had become excessively fragmented.

RESULTS

\section{The cortical layer in stratified eggs}

The cortical granules of $S$. purpuratus are irregular in shape and vary in maximum diameter from 0.6 to $1.2 \mu$ (cf. fig. 2). The average distribution of granules is about $47 \pm 5$ per $100 \mu^{2}$. In the centrifuged eggs in which these estimates were made (from photographs similar to fig. 2), it is possible that the distribution measured might have been too low, for the force required to stratify the cytoplasmic granules sufficiently to observe the cortex well $(10,000 \times$ gravity for 10 minutes $)$ caused a slight centrifugal migration of the cortical granules. This became especially noticeable in the cortical layers of clear quarters, where it was clearly seen that forces of 13,000 to $17,000 \times$ gravity for 15 minutes had somewhat depleted the 
cortex of granules on the centripetal pole. Since the heavier quarters and the centrifugal poles of the lighter quarters showed a denser distribution of granules, it is probable that at least some of the granule movement was intra-cortical.

Occasionally, however, large granules, unmistakably of cortical origin, were observed in the clear hyaline zone of the endoplasm following centrifugation at forces around 15,000 $\times$ gravity. These granules were found to be the heaviest inclusions in the cell (see below). The fact that they did not fall rapidly and more or less freely through the endoplasm indicated that they might have been attached to the centripetal cortex, suspended from a stretched membrane.

\section{Separation of cortical material from whole eggs}

Jelly-free whole eggs were layered over 0.96 molar sucrose and the centrifuge tubes tilted to mix the boundaries into density gradients. Sets of two tubes were centrifuged for from 15 to 45 minutes at speeds from 15,000 to 39,000 revolutions per minute. Since halves, quarters and smaller fragments of eggs separated into distinct layers in the density gradient, each was subjected to a different force, depending upon the angular velocity of the rotor and the radius of rotation. The minimum speed at which cortical separation was detected was 20,000 r.p.m. A few egg fragments exposed at this speed to forces of about $21,000 \times$ gravity lost patches of cortical material, and some isolated cortical granules were recovered throughout the medium and at the bottom of the tube. When eggs were exposed to $40,000 \times$ gravity and above, eggs underwent fragmentation into halves, quarters and then into smaller fragments. If the force was applied slowly, the fragmentation probably took place before cortical separation. If the force was applied rapidly, it was evident that the cortex was stripped from many eggs before much fragmentation had occurred; this was clear from the size of the cortical hulls described below (cf. fig. 3). Egg fragments that had been exposed to 60,000 $X$ gravity and above had lost all cortical material. The cyto- 
plasm of these fragments had the same appearance as that of the untreated unfertilized egg. However, several attempts at fertilizing them failed to produce any visible indications that sperm had penetrated, or that the fragments themselves had

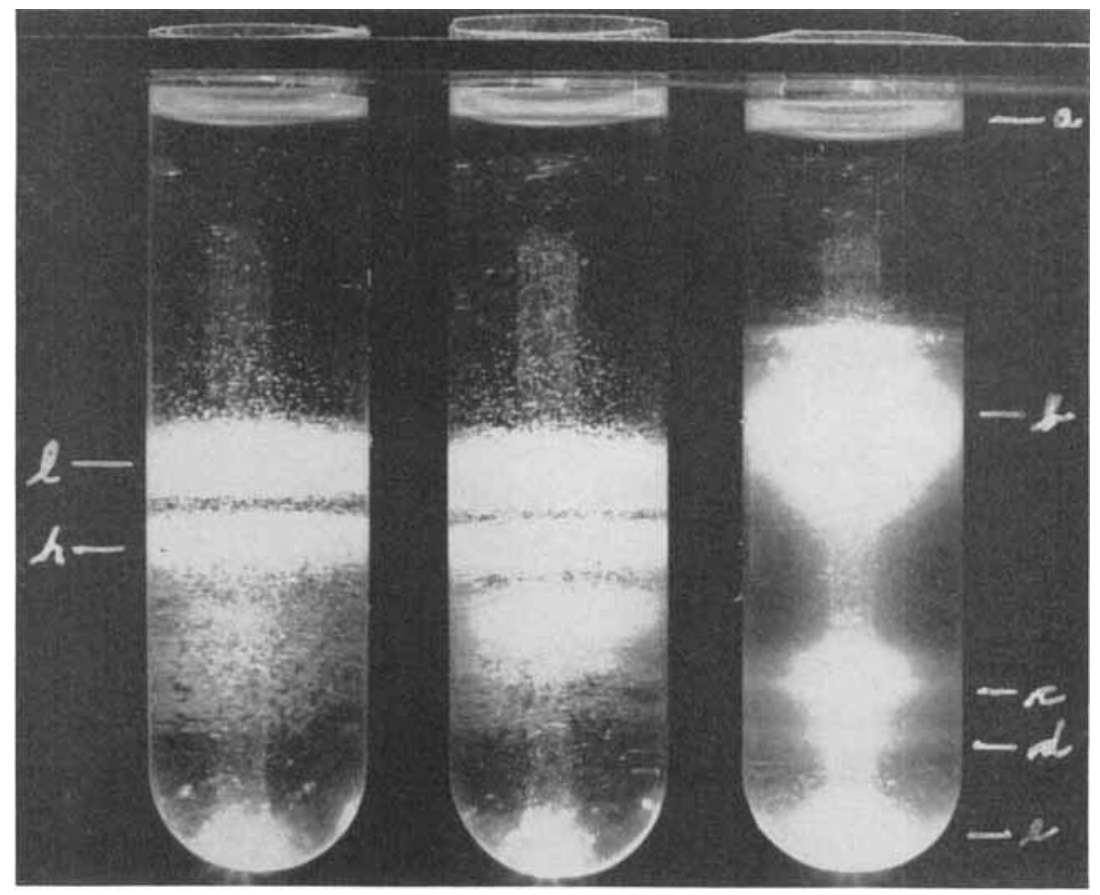

Fig. I Plastic centrifuge tubes containing centrifuged sea urchin eggs layered over isotonic sucrose. Left: (l) light and ( $h$ ) heavy halves. Middle: quarters (lighter two not well resolved). Right: after 45 minutes centrifugation at 35,000 RPM over a double gradient (see text): (a) fragments containing fat-ca]) material, (b) light fragments, (c) heavy fragments, (d) isolated cortical granules and sheets, (e) thick "membranes" and cortical hulls.

reacted to the presence of sperm. Most of the fragments were of the order of size of eighths or sixteenths of unfertilized eggs.

\section{Isolated cortical material}

1. Empty cortical hulls, almost always with granules densely packed on the centrifugal hemisphere but sparse on 
the centripetal pole, were usually not plentiful, but of sufficient size to indicate origin from half or quarter egg fragments (fig. 3).

2. Isolated "membranes," too thick to be composed of vitelline membrane material alone, were observed to contain

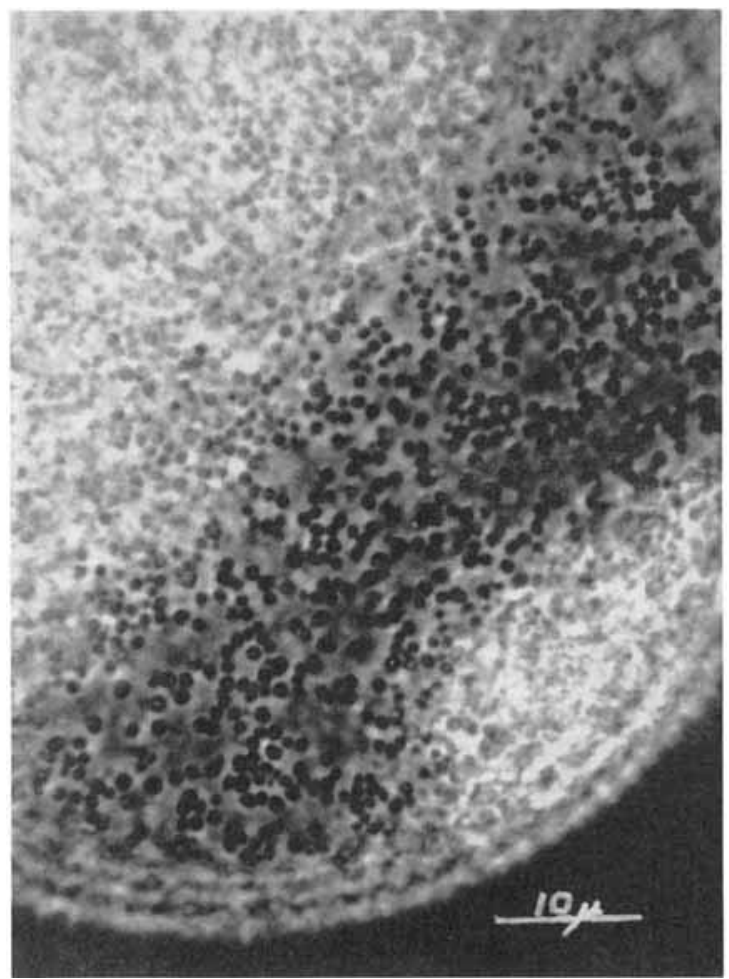

Fig. 2 S. purpusatus egg stratified by 10 minutes centrifugation at 10,000 $\times$ gravity to show granules in the cortical layer. Zeiss phase contrast objective, $100 \times$.

very small dark granules not large enough to be intact cortical granules (compare fig's. 4, 5). It seems likely that the "membranes" consisted of the vitelline membranes (or their precursors), plus "luminous hyaline layers" (cf. Allen and Hagström, '55), to which the cortical granules might once have been attached. The small granules attached to these thick 
"membranes" were probably fragments of cortical granules, for, if they existed as small granules in the cortex, they should have been observed there in the intact centrifuged egg.

3. Sheets of cortical matcrial, consisting of cortical gel containing cortical granules, were very common in the bottom sediment following centrifugation. The "membranes" (above), being heavier than the cortical material beneath, evidently became torn away from this material, leaving it isolated from its outer layer (fig. 6). Some cortical strips exhibited fibrils extending in all directions in the plane of the layer; this observation, however, was not common.

4. Isolated cortical granules were found distributed throughout the sucrose medium after brief centrifugation at forces above $21,000 \times$ gravity, but they sedimented only upon prolonged centrifugation. It is likely that most of these had been torn loose from the sheets of cortical material. Isolated cortical granules often adhered closely to ane another, showing that they carried around them little or no cortical gel; the cortical granules in cortical sheets were often more crowded than in the cortex of the normal unfertilized egg, but still were separated by discernable spaces (compare figs. $6,7)$.

5. Heavy egg fragments were found along with cortical sheets and isolated granules in the bottom fractions; they contained mostly cortical material with very little endoplasm and assumed a variety of shapes, the most common being spheres and cylinders. Upon dialysis against sea water, many of the fragments at the bottom disintegrated; of those which remained intact, many responded to the presence of spermatozoa by disintegration. Sometimes two heavy fragments were connected by long fibers which evidently consisted of extended cortical material (fig. 8).

6. Droplets of high refractive index were often seen in the bottom fraction. A few times these granules were observed to form from cortical gel undergoing cytolytic changes resulting from excessive heat from the illumination source (fig. 7). 


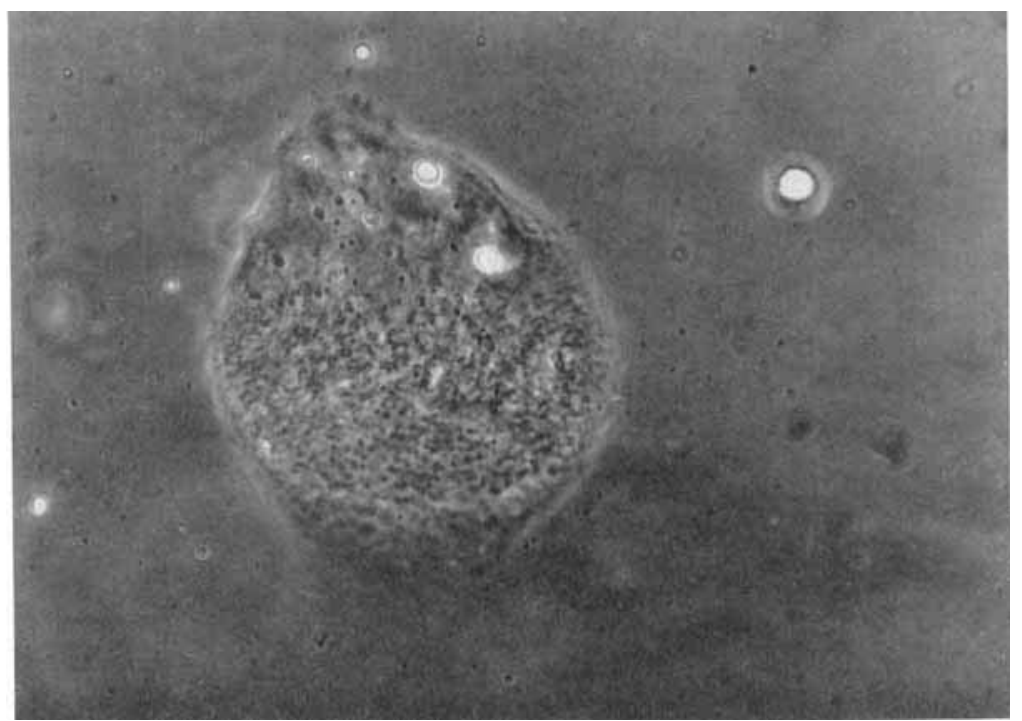

Fig. 3 A hull of cortical material. Note depletion of granules at centripetal pole. Centrifugal foree $35,000 \times$ gravity for 15 minutes.

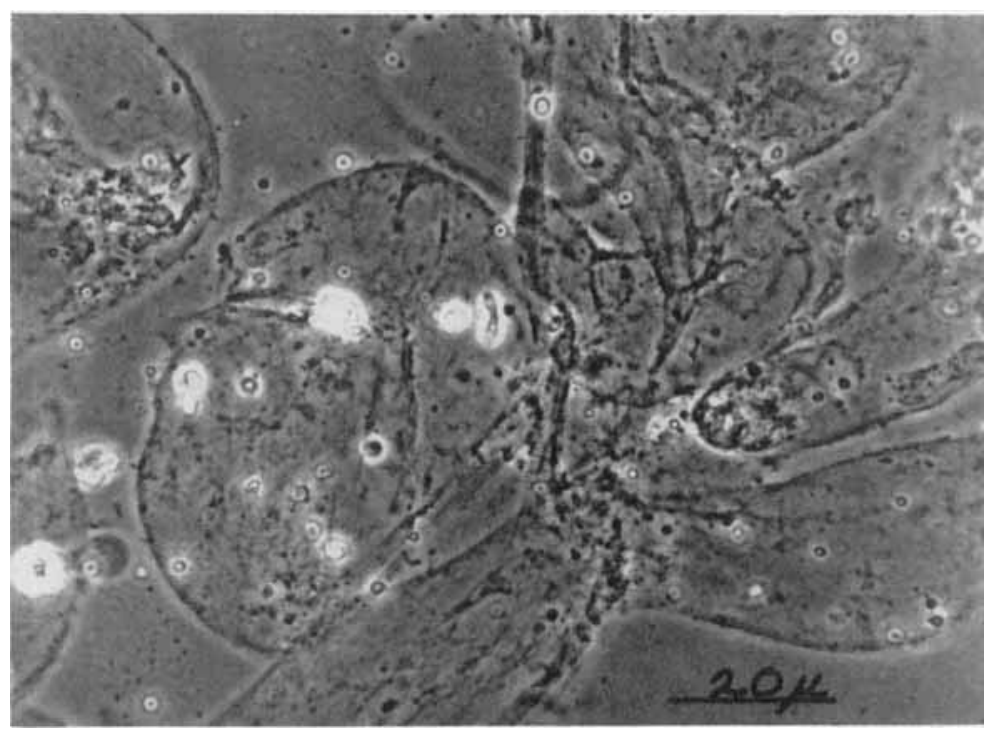

Fig. 4 Isolated "nembranes"' (see text). Obtained as in figure 4. 


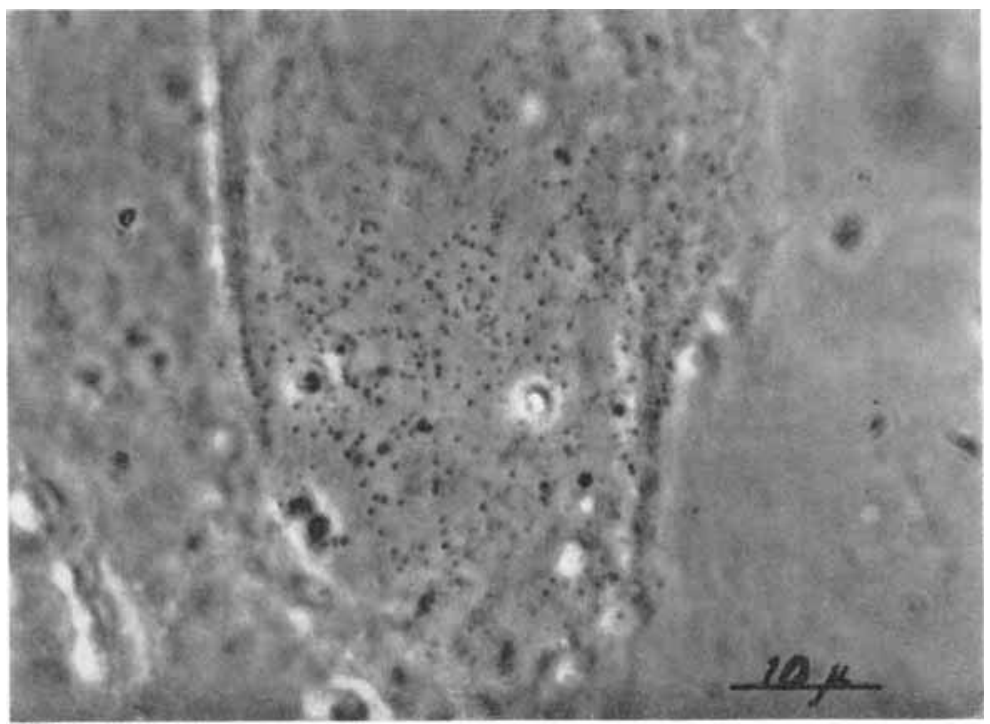

Fig. 5 Small granules believed to be cortical granule fragments attached to the isolated "membranes"' (see text).

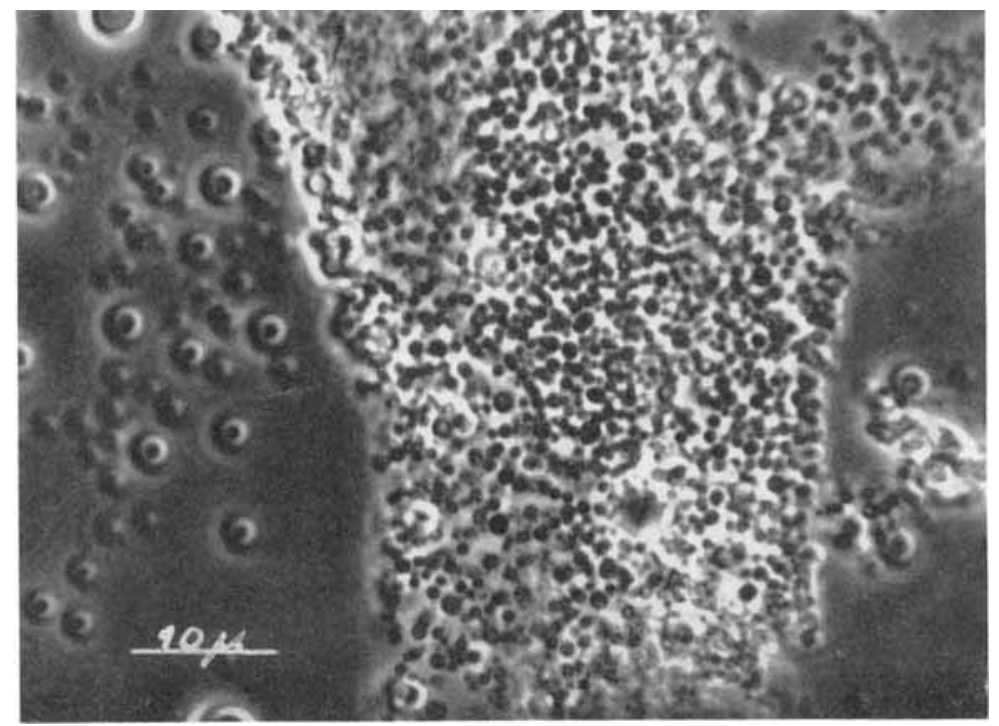

Fig. 6 A sheet of isolated cortical gel containing cortical granules probably somewhat concentrated by the effects of centrifugal force. 


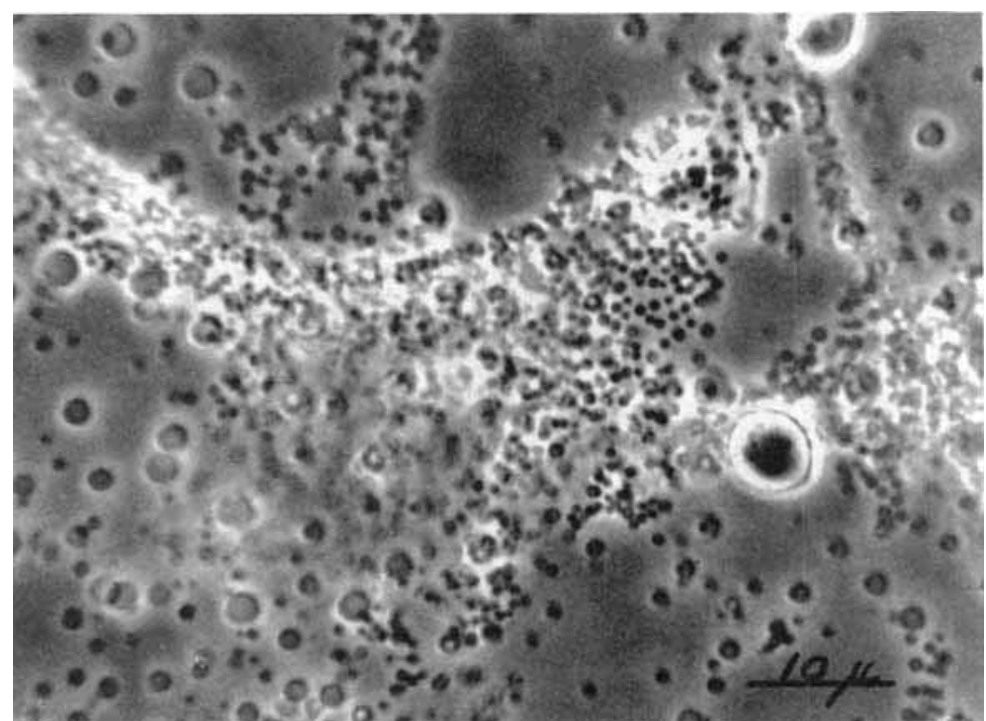

Fig. 7 Sheets of cortical material in the proeess of disintegrating; note the highly refractile droplets which form apparently as a result of cortieal disintegration.

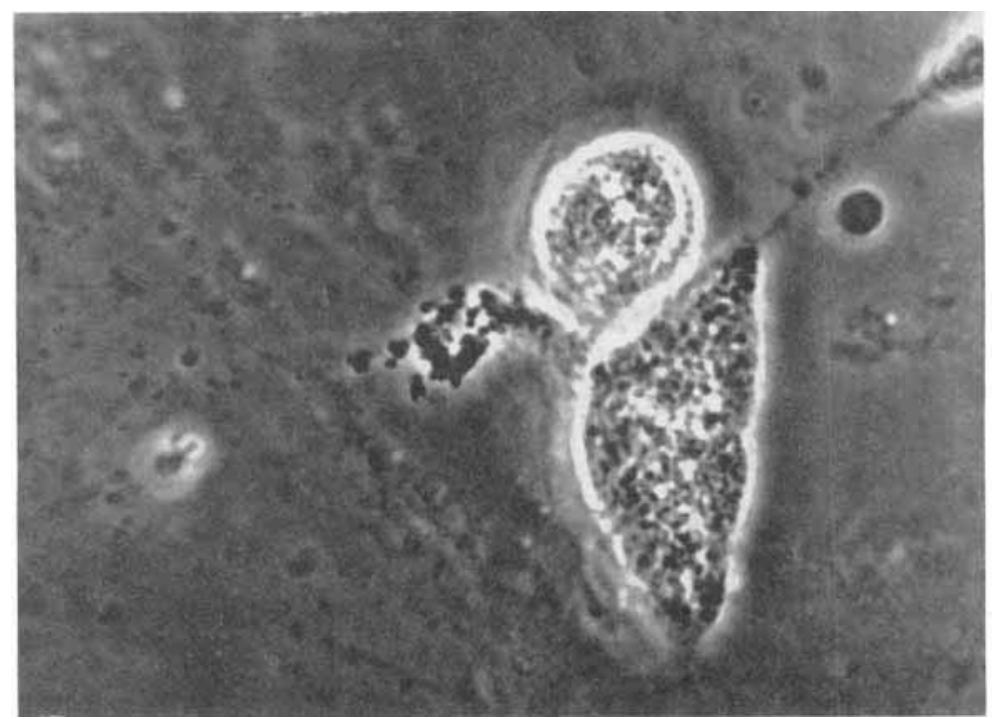

Fig. 8 Heavy egg fragments consisting mostly of cortical material with a small amount of yolky endoplasm. Note the string of cortical material attached to the larger fragment. 


\section{Separation of cortical material from egg halves and quarters}

In the process of separation of cortical material from whole eggs, one of the first effects of centrifugation is to fragment the eggs into halves and quarters. Accordingly, it was not surprising that the yield of isolated cortical material did not differ qualitatively when cortical isolation was carricd out on each of the halves and quarters of the egg. The results were different, however, with respect to the relative quantities of the different kinds of cortical material obtained. Fewer and smaller cortical hulls were obtained from centrifugation of light quarters, and heavy fragments were almost entirely lacking. It might be concluded that the purest preparations of isolated cortical material would be obtained from the light quarters alone. This procedure has the disadvantage of decreasing the yield, however, and if slight impurities consisting chiefly of yolky cytoplasm could be tolerated, it would be preferable to begin with whole eggs or light halves.

\section{Determination of the specific gravity of cortical material}

In a double density gradient (sea water over 0.96 molar sucrose, over 1.17 to 1.46 molar sucrose) it was possible to estimate the specific gravity of some of the cortical components. Isolated granules sank in concentrations of sucrose less than 1.32 molar, but remained suspended in more concentrated solutions. The specific gravity of 1.32 molar sucrose at $20^{\circ}$ is approximately 1.168 , but since the granules were somewhat shrunken in this hypertonic environment, it is probable that their specific gravity was somewhat less. Sheets of cortical material remained with the isolated granules, indicating that the cortical gel was not very different from the granules in specific gravity. Isolated "membranes", sank even in 1.75 molar sucrose, the most concentrated solution used. No concerted attempt was made to obtain high resolution between layers of cortical material. 


\section{The stability of isolated cortical material in sucrose and in sea water}

Isolated cortical material kept its structural integrity for several hours in sucrose solutions. Upon dialysis against sea water, many of the strips or hulls of cortical material became slightly "brownish." Several attempts to fertilize dialyzed cortical material did not produce evidence of response to insemination. However, it could not be proven that none of the material had responded. No attempts were made to facilitate a response to insemination by the addition of favoring substances.

\section{DISCUSSION}

The removal of the cortex without dispersing the cell contents constitutes very strong evidence for the presence of a subcortical membrane as postulated by Parpart and Laris ('54) and by Allen ('55). The presence of such a sub-cortical membrane might have significanee in determining the mechanism of the propagation of the cortical reaction, especially as this membrane appears to be the plasma-membrane (Parpart and Laris, 54). Alterations of the permeability of this membrane might influence not only cortical conduction, but also cortico-endoplasmic interchange after the cortical reaction, when the "fertilized eortex" apparently "induces" the migration of pigment granules to a subcortical location (Allen and Rowe, '55).

It is interesting to note that E. B. Harvey ('34) first observed that the hyaline layer, which is a major part of the cortex of the fertilized egg, was stripped from the surface of the sea urchin egg by moderate centrifugal forces. The present writer, in unpublished experiments with Arbacia eggs, has found that pretreatment of unfertilized eggs with chymotrypsin ( $0.10 \%$ for 15 minutes) permits subsequent isolation by centrifugation of hyaline layers in considerable quantity.

The structure of isolated cortical material may provide clues to the structure of the surface layers of the intact egg. 
In a previous report on the isolation of cortex by stripping eggs attached to glass (Allen, '55), it appeared likely that the entire surface of the egg was removed and isolated (i.e. the cortical gel layer with its granules, the luminous hyaline layer and the vitelline membrane or its precursor). In the present experiments, all of these components seem to have remained together only in the cortical hulls, which were the least common of the forms of isolated cortical material. The isolated "membranes," which constituted the heaviest fraction of cortical material, probably became torn away from the inner cortical gel layer through a difference of specific gravity. These "membranes" almost certainly contained more than the vitelline membrane or its precursor, for the vitelline membrane of the unfertilized egg is extremely thin; in fact, there is some doubt that it exists as a membrane on the egg surface prior to fertilization (cf. Allen and Hangström, '55). The thickness of the isolated "membranes" and the fact that they contained small granules indicated that another inner layer probably accompanied the vitelline membrane. This must have been the "luminous hyaline layer," and the small granules must have been fragments torn from cortical granules attached in this layer. Such an attachment would partly explain (1) the resistance of cortical granules to stratification under centrifugal force, and (2) the suspension of cortical granules which were pulled into the hyaline endoplasmic zone of the unfertilized centrifuged egg.

The cortical gel layer, which was isolated in sheets or strips of material, must possess considerable rigidity of its own to withstand the shearing forces that must have been imposed by the tearing away of its outer layers. The observation of fibrils extending from strips of cortical gel might indicate a structural basis for the observed cortical rigidity. The failure of fibrils to appear in all preparations of isolated cortex might mean either that (1) the fibrils themselves were artifacts produced by sucrose, centrifugal force or a state of isolation from the rest of the cell, or (2) that the fibrils had been destroyed in most cases by one of the above treatments. 
Although it is not possible to decide definitely which of these possibilities is correct, the latter seems more probable, for it was observed that strips of cortex isolated by pulling the egg away from adhering glass surfaces nearly all possessed such fibrils. Moreover, the cortex isolated in this manner was capable of response to insemination (Allen, 55). The phenomenon of the formation of highly refractive droplets from disintegrating cortical gel is interesting and possibly important, but its mechanism and significance are not entirely clear.

The possibilities of the method described in this paper for the investigation of the chemistry of the cortex seem preferable to those offered by the method of Motomura ('54). The present writer hopes to return to this problem at the earliest opportunity. It is unusually good fortune when cellular structure provides the possibility of isolating a component (even though "extracellular") without serious danger of impurity through direct physical contamination or adsorption of enzymes or other substances from the ground substance of the cell. The present method at least promises that what is recovered in the sediment will be cortical material, although the fact that this material does not appear to be physiologically active may indicate that something has been lost on isolation.

This study indirectly points to the importance of the cortex for fertilization. As Chambers ('21) showed by microdissection studies, egg fragments without cortical material do not respond to insemination.

Interesting as they may be, studies on the kineties of the cortical change alone, or of the physiological effects of the cortical change will not elucidate its mechanism in molecular terms. Direct chemical investigations of the egg cortex are required before the mechanism of the cortical reaction can be properly elucidated.

\section{SUMMARY}

A method is described for the isolation of cortical material from the egg of the sea urchin, Strongylocentrotus purpuratus, 
by centrifugation. Separation of cortical material from the rest of the egg was made possible by a large specific gravity difference between the lighter egg fragments and the heavy cortical layer. This was accomplished without disruption of the cell fragments and without dispersion of the endoplasm of the cells from which the cortical material was removed.

Isolated cortical material in the form of (1) empty hulls of cortical material, (2) isolated "membranes," actually consisting of the vitelline membrane or its precursor plus the luminous hyaline layer, (3) sheets or strips of cortical gel layer (torn away from the outer membranous layers), (4) isolated single cortical granules devoid of cortical gel material, and (5) heavy egg fragments consisting almost entirely of cortical material. These fragments were the only ones containing any endoplasmic material, and they could be eliminated by selecting only the light quarters for subsequent isolation of cortical material.

Egg fragments deprived of cortical material did not respond to insemination when returned to sea water; isolated cortical material for the most part failed to respond as far as could be determined. However, the heavy fragments described above were occasionally seen to disintegrate upon return to sea water or in the presence of spermatozoa.

The possible significance of isolated cortical material is discussed in relation to the structure of the surface layers of the egg and the mechanism of the cortical reaction.

I am very much indebted to Dr. Daniel Mazia for the pleasure and privilege of performing this work in his laboratory in the Department of Zoology of the University of California, Berkeley.

\section{LITERATURE CITED}

ALLEN, R. D. 1955 The fertilization reaction in isolated cortical material from sea urchin eggs. Exptl. Cell Res., 8: 397-399.

Allen, R. D., AND B. E. Hagströn 1955 Some interrelationships among cortical reaction phenomena in the sea urchin egg. Exptl. Cell Res. Suppl., 3: 1-15. 
Allen, R. D., AND E. C. Rowe 1955 Pigment migration in partially fertilized Arbacia eggs. Biol. Bull., 109(3): 344-345.

Chambers, R. 1921 Mierodissection studies, III. Some problems in the maturation and fertilization of the echinoderm egg. Biol. Bull., 41: 318-350.

HARVEY, E. B. 1932 The development of half and quarter eggs of Arbacia punctulata and of strongly eentrifuged whole eggs. Biol. Bull., 62( 2$): 155-167$.

1934 Effects of centrifugal force on the ectoplasmic layer and nuclei of fertilized sea urchin eggs. Biol. Bull., 66(2): 228-245.

HaRVEY, F. N. 1911 Studies on the permeability of cells. J.E.Z., 10: $507-556$.

Moser, F. 1939 Studies on a cortical layer response to stimulating agents in the Arbacia egg. I. Response to insemination. J.E.Z., 80 : $423-440$.

Morontera, I. 1954 On a metlod of isolation of the egg cortex in the sea urchin egg. Sci. Rep. Tôhoku Imp. Univ. Sendai (Biol.), 20: 318-321.

PARpart, A. K., AND P. C. LARIS 1954 Where is the plasma membrane of the unfertilized egg of Arbacia punctulata? Biol. Bull., 10\%: 301.

Runnström, J. 1923 Eine Lipoide Oberfläehenschicht bei dem Seeigelei. Acta Zool., 4: 285-311. 